\title{
INFLUENCE OF SILICON ON QUALITY FACTOR, MOTIONAL IMPEDANCE AND TUNING RANGE OF PZT-TRANSDUCED RESONATORS \\ Hengky Chandrahalim ${ }^{1}$, Sunil A. Bhavel, Ronald Polcawich ${ }^{2}$, Jeff Pulskamp ${ }^{2}$, Daniel Judy ${ }^{2}$, Roger Kaul ${ }^{2}$ and Madan Dubey ${ }^{2}$ \\ ${ }^{1}$ OxideMEMS Laboratory, Cornell University, Ithaca, NY 14853, USA \\ ${ }^{2}$ US Army Research Laboratory, Adelphi, MD 20783, USA
}

\begin{abstract}
This paper provides a quantitative comparison and explores the design space of PZT-only (Lead Zirconium Titanate) and PZT-onSilicon length-extensional mode resonators for incorporation into RF MEMS filters and oscillators. We experimentally measured the correlation of motional impedance $\left(R_{X}\right)$ and quality factor $(Q)$ with the resonators' silicon layer thickness $\left(t_{S i}\right)$. For identical lateral dimensions and PZT-layer thickness $\left(t_{P Z T}\right)$, the PZT-on-Silicon resonator has higher resonant frequency (dominated by silicon), higher $Q(5,100$ vs. 140$)$ and lower motional impedance ( $51 \Omega$ vs. $205 \Omega$ ). However, PZT-only resonator demonstrated much wider frequency tuning range $(5.1 \%$ vs. $0.2 \%)$.
\end{abstract}

\section{INTRODUCTION}

Numerous applications in wireless communications and sensor networks have motivated the development of on-chip, high- $Q$ MEMS resonators and filters to realize portable radios that consume low power and operate at the global range of frequency standards. Dielectrically transduced thickness shear mode and contour-mode resonators have the capability to reach high frequency of operation while maintaining high $Q$ and low motional impedance [1,2]. A band-pass filter can be constructed by electrically or mechanically coupling an array of these resonators. However, the inadequate effective coupling efficiency of the dielectrically transduced resonators limits the bandwidth of the filter, creating a demand for transducers with high electromechanical coupling coefficient.

Aluminum Nitride (AIN) is a popular material for fabrication of thin film bulk acoustic wave (BAW) and contour-mode resonators and filters because it possesses a high acoustic velocity, high quality factor and post-CMOS integration capability $[3,4]$. However, intermediate frequency applications have driven the investigations for new piezoelectric/ferroelectric materials for resonators. At low frequencies, ferroelectrics like PZT are better suited as they avoid thick film requirements and reduce the area required for the filters. Furthermore, PZT exhibits larger electromechanical coupling coefficient than AlN, enabling the design of larger $\%$ bandwidth filters [5].

A resonator with dynamically tunable center frequency will not only overcome fabrication tolerances and thermal drift, but will also facilitate filter design that has the ability to discern bandwidths between $0.1 \mathrm{MHz}$ to $5 \mathrm{MHz}$, enabling a handheld analog spectral processor with voltage dependent dynamic frequency trimming and tuning. PZT has been previously shown to have frequency tuning capabilities in [6,7]. However, PZT-only resonators are well known to have low quality factor $(Q<300)$ $[5,8]$. In order to overcome the low quality factor of PZT-only resonators, we developed a new fabrication technology to integrate PZT transduction with single-crystal silicon resonators.

\section{LENGTH-EXTENSIONAL MODE RESONATORS}

Previous results have shown that length-extensional mode vibrations can be excited by sandwiching a piezoelectric transducer (AlN, Zinc Oxide, PZT, etc.) between metal electrodes $[4,9]$.

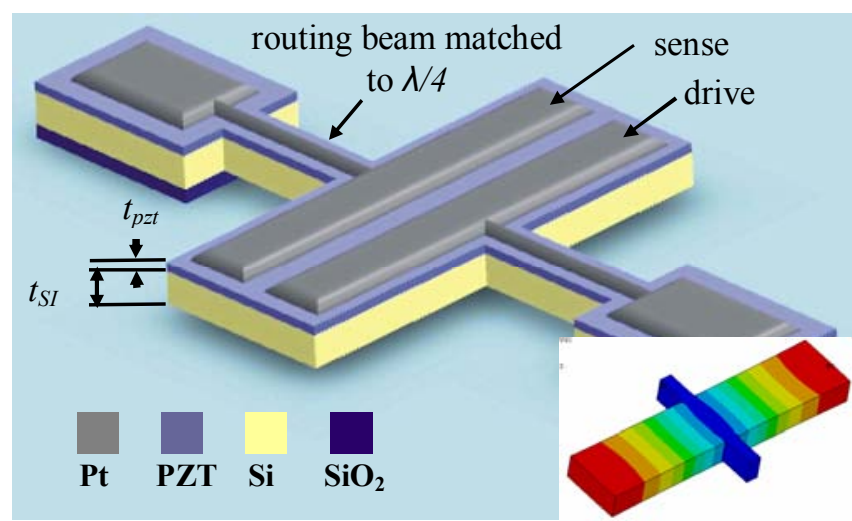

Figure 1: 3D schematic of PZT-on-silicon length-extensional mode resonator, and (inset) ANSYS mode shape.

However due to poor crystallinity and repeatability of the piezoelectric thin-films, several research groups have chosen to use the piezoelectric material for actuation and sensing, while utilizing single-crystal silicon (SCS) as the resonating structure $[10,11]$ (Figure 1). In this effort, PZT transduced resonators were fabricated with and without a $10 \mu \mathrm{m}$ thick silicon device layer to explore the insertion loss and $Q$ trade-offs between the two types of resonators. The frequency independent motional impedances of the fundamental mode of PZT transduced length-extensional mode two-port resonators are

$$
\begin{aligned}
& R_{X_{-} P Z T-o n-S O I}=\frac{\pi}{2} \cdot \frac{\sqrt{E_{E f f} \cdot \rho_{E f f}}}{Q} \cdot\left(\frac{t_{S i}+t_{P Z T}}{E_{P Z T}{ }^{2} \cdot W \cdot d_{31}{ }^{2}}\right) \ldots \\
& R_{X_{-} P Z T-o n l y}=\frac{\pi}{2} \cdot \frac{\sqrt{E_{P Z T} \cdot \rho_{P Z T}}}{Q} \cdot\left(\frac{t_{P Z T}}{E_{P Z T}{ }^{2} \cdot W \cdot d_{31}{ }^{2}}\right) \ldots
\end{aligned}
$$

where $\rho_{E f f}$ and $E_{E f f}$ are the effective (PZT + silicon) density and Young's modulus, $W$ is the resonator width, $d_{31}$ is the transverse piezoelectric coefficient, and $\rho_{P Z T}$ and $E_{P Z T}$ are the density and Young's modulus of the PZT thin-film. PZT is acoustically lossy material resulting in PZT-only resonators having low $Q$. Integrated PZT transduction with single-crystal silicon resonators retains the mechanical energy within the high quality silicon device layer. Equation 1 indicates that there will be a net decrease in $R_{X}$ if the improvement in $Q$ outweighs the increase in $t_{S i}$ for PZT-on-silicon resonators. However, due to the silicon carrier layer, the PZT-onsilicon resonators have to perform extra work in order to overcome the inertia of the proof-mass. Hence the PZT-only resonators will exhibit higher pole-zero separation than PZT-on-silicon resonators in a one-port configuration. Therefore by changing $t_{S i}$ we can tradeoff between narrow bandwidth and wide bandwidth filters.

\section{FABRICATION PROCESS}

The fabrication process flow for the PZT-on-silicon resonators is shown in Figure 2. We started with an SOI wafer with $10 \mu \mathrm{m}$ 
I.

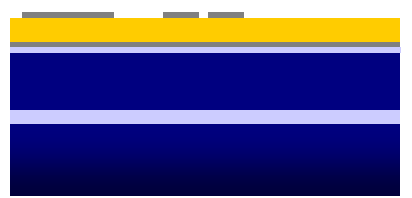

II.
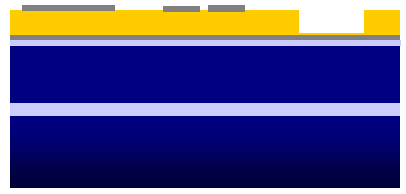

III.

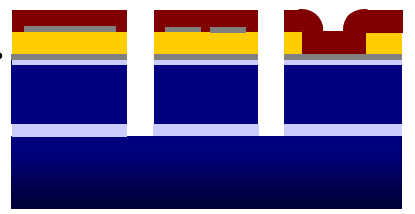

IV.

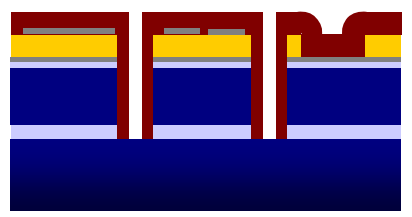

V.

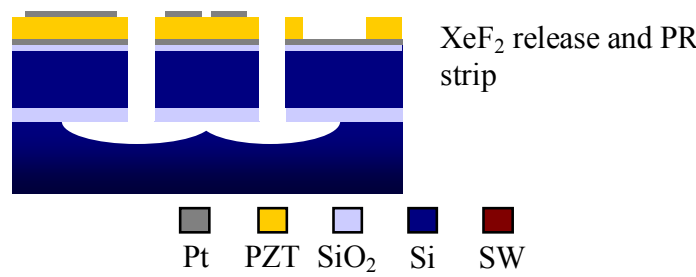

Figure 2: Fabrication process-flow for PZT-on-Silicon resonators. By replacing the SOI wafer with a Silicon wafer, we used the same fabrication process to manufacture PZT-only resonators.
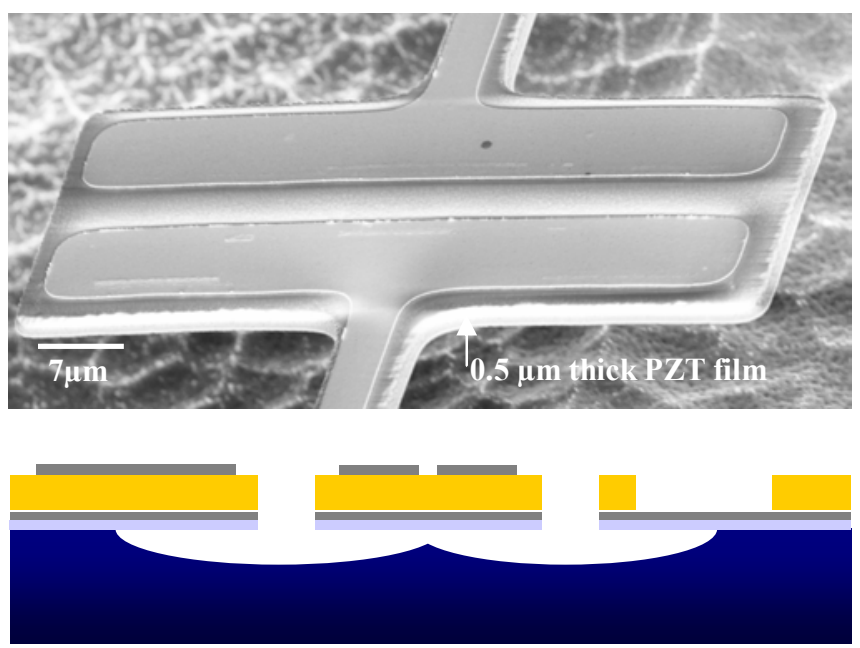

$\begin{array}{llll} & & & \\ \mathrm{PZT} & \mathrm{SiO}_{2} & \mathrm{Si}\end{array}$

Figure 3: SEM and process cross-section of a released PZT-only resonator. The cross-section is drawn in such a way as to identify the signal and ground pads, the drive and sense electrodes and the released resonator.

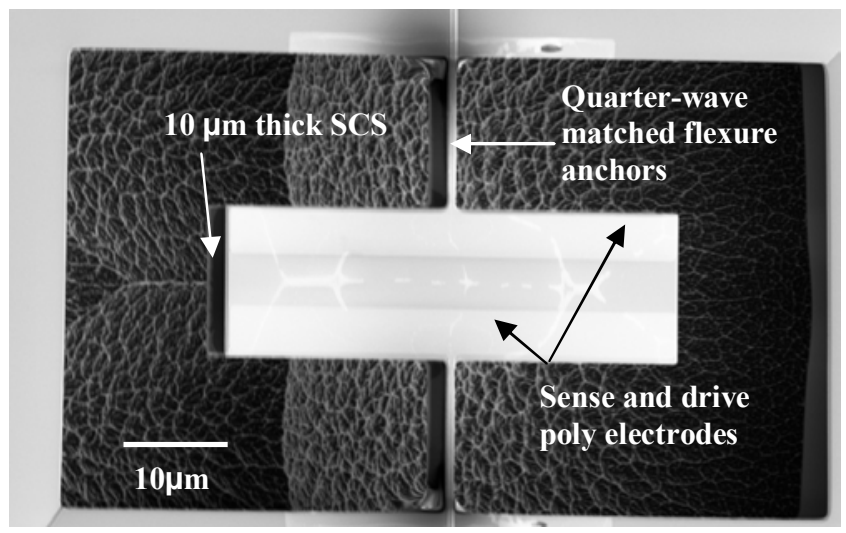

Figure 4: SEM of released PZT-on-silicon resonator. The isotropic pit due to the $\mathrm{XeF}_{2}$ etch can be seen in the background.

thick silicon device layer. We deposited $50 \mathrm{~nm} \mathrm{SiO}_{2}$ to mitigate lattice mismatch issues between the PZT-metal stack and the silicon. Then we successively deposited $100 \mathrm{~nm}$ platinum bottom metal, $0.5 \mu \mathrm{m}$ PZT [12] and another $100 \mathrm{~nm}$ platinum top metal. We patterned the top metal electrodes and signal pads using lift-off followed by pattern and wet-etch of PZT to open ground pads. The resonator was then defined by anisotropic etching of the PZT, platinum, silicon dioxide, silicon device layer and the buried oxide. A novel technique of dry releasing the devices using a gentle frontside $\mathrm{XeF}_{2}$ alleviated the need for backside DRIE used in [13]. Figures 3 and 4 show PZT-only and PZT-on-silicon bar resonators fabricated in this process.

\section{MEASUREMENT RESULTS}

The resonators were characterized after performing SOLT calibration followed by SOT de-embedding. Measured transmission response of the PZT-only and PZT-on-silicon resonators with the exact same lateral dimensions in air at room temperature and pressure are shown in Figure 5. The PZT-onsilicon resonators exhibit higher $Q$, higher resonant frequency and lower motional impedance compared to their PZT-only counterparts. These measurement results confirm that integrating

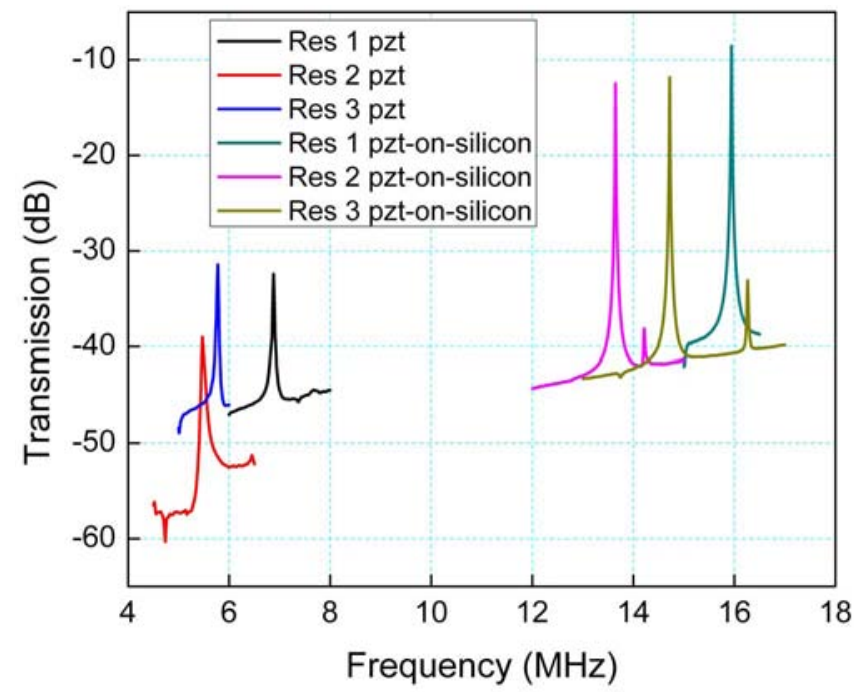

Figure 5: Measured transmission response of PZT-only and PZTon-Silicon resonators with the exact same lateral dimensions in air at room temperature and pressure. 
PZT transduction with single-crystal silicon retains the mechanical energy within the high quality silicon device layer. The improvement in $Q(35 \times)$ outweighs the increase in $t_{S i}(10 \mu \mathrm{m})$, resulting in a net decrease in $R_{X}$ (Tables 1 and 2).

Table 1: The measured characteristics of PZT-only resonators.

\begin{tabular}{|l|l|l|l|}
\hline & Res 1 PZT & Res 2 PZT & Res 3 PZT \\
\hline $\begin{array}{l}\text { Dimensions } \\
\left(\mu \mathrm{m}^{2}\right)\end{array}$ & $240 \times 40$ & $280 \times 60$ & $260 \times 60$ \\
\hline$Q$ & 202 & 91 & 241 \\
\hline$f_{\text {CENTER }}(\mathrm{MHz})$ & 6.88 & 5.47 & 5.78 \\
\hline$R_{X}(\Omega)$ & 4,078 & 8,812 & 3,628 \\
\hline
\end{tabular}

Table 2: The measured characteristics of PZT-on-SOI resonators.

\begin{tabular}{|l|l|l|l|}
\hline & $\begin{array}{l}\text { Res 1 PZT- } \\
\text { on-silicon }\end{array}$ & $\begin{array}{l}\text { Res 2 PZT- } \\
\text { on-silicon }\end{array}$ & $\begin{array}{l}\text { Res 3 PZT- } \\
\text { on-silicon }\end{array}$ \\
\hline $\begin{array}{l}\text { Dimensions } \\
\left(\mu \mathrm{m}^{2}\right)\end{array}$ & $240 \times 40$ & $280 \times 60$ & $260 \times 60$ \\
\hline$Q$ & 5,040 & 3,820 & 4,224 \\
\hline$f_{C E N T E R}(\mathrm{MHz})$ & 15.94 & 13.65 & 14.72 \\
\hline$R_{X}(\Omega)$ & 167 & 319 & 290 \\
\hline
\end{tabular}

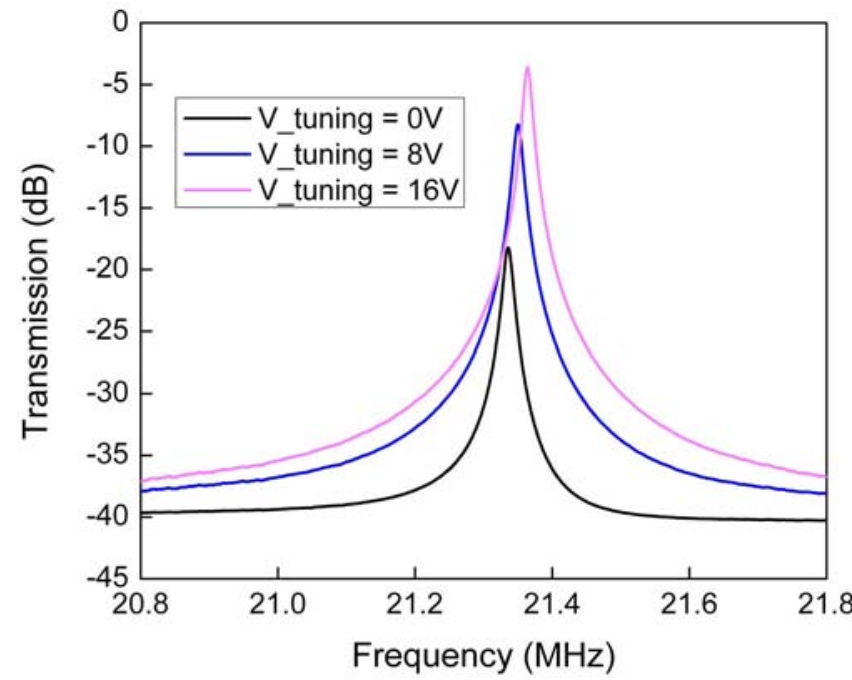

Figure 6: Measured transmission of a $190 \mu \mathrm{m} \times 40 \mu \mathrm{m}$ PZT transduced resonator on $10 \mu \mathrm{m}$ silicon with different bias voltages in air at room temperature and pressure. $0.2 \%$ frequency tuning capability is observed due to DC bias dependent Young's modulus and density of PZT thin film. (only 3 transmission plots are shown to avoid crowding).

Table 3: The measured characteristics of PZT-on-SOI resonator with five different bias voltages.

\begin{tabular}{|l|l|l|l|l|l|}
\hline $\begin{array}{c}\text { Tuning } \\
\text { Voltage }\end{array}$ & $0 \mathrm{~V}$ & $4 \mathrm{~V}$ & $8 \mathrm{~V}$ & $12 \mathrm{~V}$ & $16 \mathrm{~V}$ \\
\hline$Q$ & 2,020 & 2,021 & 2,021 & 2,022 & 2,023 \\
\hline$f_{C}(\mathrm{MHz})$ & 21.332 & 21.340 & 21.345 & 21.355 & 21.365 \\
\hline$R_{X}(\Omega)$ & 582 & 223 & 142 & 83 & 50 \\
\hline
\end{tabular}

\section{VOLTAGE TUNING}

Wang et al and Cho et al have demonstrated that PZT has a DC-bias dependent Young's modulus, density and permittivity $[6,7]$. As DC bias increases, the increase in the piezoelectric coefficient, $d_{31}$, and decrease in permittivity leads to an overall increase in the electromechanical coupling coefficient and decrease in $R_{X}$. By taking an advantage of the DC bias dependent piezoelectric coefficient $d_{3 l}$, we can compensate insertion loss of the resonators due to air damping, anchor losses and fabrication errors. The measured transmission of a $190 \mu \mathrm{m}$ x $40 \mu \mathrm{m}$ PZT transduced resonator on $10 \mu \mathrm{m}$ silicon with different bias voltages is shown in Figure 6. A $0.2 \%$ frequency tuning capability is observed due to the DC bias dependent Young's Modulus of PZT thin film. Since the resonant frequency is dominated by the silicon device layer, there is very little resonant frequency tuning, whereas the improvement in electromechanical coupling leads to $11 \times$ improvement in motional impedance at $16 \mathrm{~V}$ DC bias. The measured characteristics of the resonator are summarized in Table3.

In contrast, the measured transmission of a $90 \mu \mathrm{m} \times 20 \mu \mathrm{m}$ PZT-only resonator with different bias voltages in air at room temperature and pressure is shown in Figure 7. The PZT-only resonators demonstrate $5.1 \%$ DC bias dependent frequency tuning capability, the highest tuning range reported by any piezoelectrically transduced contour mode resonators to date. The measured characteristics of the resonator are summarized in Table4.

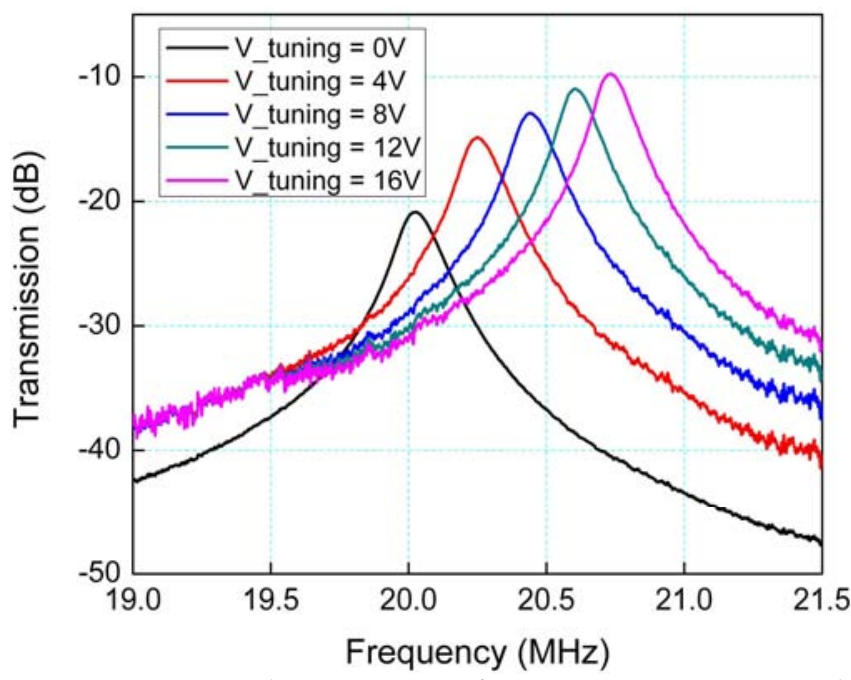

Figure 7: Measured transmission of a $90 \mu \mathrm{m} \times 20 \mu \mathrm{m}$ PZT-only resonator with five different bias voltages in air at room temperature and pressure. 5.1\% frequency tuning capability is observed due to DC bias dependent Young's modulus and density of PZT thin film.

Table 4: The measured characteristics of PZT-only resonator with different bias voltages.

\begin{tabular}{|l|l|l|l|l|l|}
\hline $\begin{array}{l}\text { Tuning } \\
\text { Voltage }\end{array}$ & $0 \mathrm{~V}$ & $4 \mathrm{~V}$ & $8 \mathrm{~V}$ & $12 \mathrm{~V}$ & $16 \mathrm{~V}$ \\
\hline$Q$ & 125 & 126 & 136 & 147 & 148 \\
\hline$f_{C}(\mathrm{MHz})$ & 20.025 & 20.250 & 20.440 & 20.610 & 20.730 \\
\hline$R_{X}(\Omega)$ & 1,004 & 453 & 343 & 253 & 207 \\
\hline
\end{tabular}




\section{CONCLUSION}

We have fabricated and characterized DC field dependent PZT transduced length-extensional mode resonators with and without silicon device layer. By varying the silicon thickness (thereby the \% mass of crystalline silicon in the resonator) and DC bias voltage we can define the desired $Q$ and frequency tuning range of the resonators, and tradeoff between steep-walled narrowbandwidth filters, low- $Q$ wide-bandwidth filters and center frequency agility.

\section{ACKNOWLEDGEMENTS}

The authors wish to acknowledge the assistance and support of Joel Martin and Brian Power of General Technical Services and Richard Piekarz from the ARL for their hard work with device fabrication.

\section{REFERENCES}

[1] H. Chandrahalim, D. Weinstein, L. F. Cheow and S. A. Bhave, "Channel-select micromechanical filters using high- $\kappa$ dielectrically transduced MEMS resonators," MEMS 2006, Istanbul, Turkey, January 22-26, 2006, pp. 894-897.

[2] H. Chandrahalim and S. A. Bhave, "Digitally-tunable MEMS filter using mechanically-coupled resonator array," $M E M S$ 2008, Tucson, Arizona, January 13-17, 2008, pp. 1020-1023.

[3] R. C. Ruby, A. Barfknecht, C. Han, Y. Desai, F. Geefay, G. Gan, M. Gat, and T. Verhoeven, "High-Q FBAR filters in a wafer-level chip-scale package," ISSCC 2002, San Francisco, California, February 3-7, 2002, pp. 184-185.

[4] G. Piazza, P. J. Stephanou and A.P. Pisano, "Single-chip multiple-frequency AIN MEMS filters based on contourmode piezoelectric resonators," JMEMS 16 (2) 2007, pp. 319-
$-328$.

[5] J. D. Larson, S. R. Gilbert and B. Xu, "PZT material properties at UHF and microwave frequencies derived from FBAR measurements," Ultrasonics Symposium 2004, Montreal, Canada, August 24-27, 2004, pp. 173-177.

[6] Q. Wang, et al, "Effect of DC bias field on the complex materials coefficients of piezoelectric resonators," Sensors and Actuators A: Physical 109 (1-2) 2003, pp. 149-155.

[7] J. Cho, et al, "Optimization of electromechanical coupling for a thin-film PZT membrane: II. Experiment," Journal of Micromechanics and Microengineering 15 (10) 2005, pp 1804-1809.

[8] B. Piekarski, D. DeVoe, M. Dubey, R. Kaul and J. Conrad, "Surface micromachined piezoelectric resonant beam filters," Sensors and Actuators A: Physical 91 (3) 2001, pp. 313-320.

[9] B. Antkowiak, et al, "Design of a high-Q, low-impedance, GHz-range piezoelectric MEMS resonator," Transducers'03, Boston, Massachusetts, June 8-12, 2003, pp. 841-846.

[10] S. Humad, R. Abdolvand, G. K. Ho, G. Piazza and F. Ayazi, "High frequency micromechanical piezo-on-silicon block resonators," IEDM 2003, Washington DC, December 8-10, 2003, pp. 957-960.

[11] A. Jaakkola, et al, "Piezotransduced single-crystal silicon BAW resonators," Ultrasonics Symposium 2007, New York City, New York, October 28-31, 2007, pp. 1653-1656.

[12] S. Dey, K. Budd, and D. Payne, "Thin-film ferroelectrics of PZT of sol-gel processing," TUFFC 35 (1) 1988, pp. 80-81.

[13] G. Ho, R. Abdolvand and F. Ayazi, "High order composite bulk acoustic resonators," MEMS 2007, Kobe, Japan, January 21-25, 2007, pp. 791-794. 